\title{
TOTAL HEALTH: Toward Continuous Personal Monitoring
}

\author{
Wenyao $\mathrm{Xu}^{1}$ and Ming-Chun Huang ${ }^{2}$ \\ 1 Department of Computer Science and Engineering University at Buffalo, \\ The State University of New York (SUNY), NY 14260-2500, USA \\ wenyaoxu@buffalo.edu \\ 2 Department of Electrical Engineering and Computer Science at Case Western \\ Reserve University, OH 44106, USA \\ ming-chun. huang@case.edu
}

\begin{abstract}
Ubiquitous personal monitoring of health is being rapidly developed in recent years. However the concept of pervasive computing in healthcare focuses on individual systems. We propose that Total Health is the transformation of healthcare that aims for complete $24 / 7$ coverage in which no one system can provide. We discuss what are the challenges that come with bringing technology into widespread use. Following this discussion, a selection of current applications in healthcare is presented. These applications use state-of-the-art systems that relate to personal health monitoring with the goal of providing a $24 / 7$ picture of one's health. The key requirements of these systems is that they provide continuous available monitoring, must be as unobtrusive as possible, require as little human intervention as possible, identify and alert health risks, and be cost effective.
\end{abstract}

\section{The Total Health Concept}

We begin by defining a number of important concepts and then we describe what Total Health means.

Firstly, Eysenbach [1] defined e-health as:

\begin{abstract}
An emerging field in the intersection of medical informatics, public health and business, referring to health services and information delivered or enhanced through the Internet and related technologies. In a broader sense, the term characterizes not only a technical development, but also a state-of-mind, a way of thinking, an attitude, and a commitment for networked, global thinking, to improve health care locally, regionally, and worldwide by using information and communication technology.
\end{abstract}

This definition of e-health places the practice of healthcare in-line with the use of electronic systems that is not just limited to Internet technology. For example, e-health can mean the application of electronic health record systems in hospitals, i.e. hospital information systems (HIS), radiological information systems (RIS), picture archive and communications systems (PACS). It in- 
cludes primary care office computerized systems, electronic prescriptions and order entry, and even email systems. Furthermore, telemedicine has been developed significantly such as in teleradiology and telepathology [2, and new applications for remote vital signs monitoring are now seeing increasing favor.

Secondly, we discuss ubiquitous computing, also known as pervasive computing. Mark Weiser first coined the term ubiquitous computing and states the following at the beginning of his influential work [3]:

The most profound technologies are those that disappear. They weave themselves into the fabric of everyday life until they are indistinguishable from it.

His vision listed a number of properties which are summarized here. Service is present everywhere. This is the idea of ubiquity, meaning everywhere. A device can either be wearable, or other devices can be available wherever you go. It is not the device but an environment. It is not a single device, but a collection of devices that work together to form a service and create the system environment. The user is not conscious of the device being used. In contrast with smartphones or laptops that demand the attention of the user, ubiquitous computing devices work for the user in the background. The user, although aware that nearby devices exist, need not actively interact with the devices nor need to worry that the services of the devices are running.

So we are now in a position to state what Total Health means:

Total Health strives for complete 24/7 coverage of well-being through the use of ubiquitous computing that focuses on e-Health monitoring, diagnosis, alerting, and action.

It is the round-the-clock environment of health management and technology utilization with as little human intervention as possible. Devices are required to be as unobtrusive as possible. This means that wireless solutions are preferable over wired solutions. Non-contact sensing is preferred over contact sensing. It is as if technology is not present at all, yet information is gathered and analyzed, results concluded, and a plan of action advised. No one system can provide complete 24-hour coverage, however, the Total Health concept strives for this using complementary systems. Total Health aims for anytime and anywhere health assurance. In later sections, we describe current technology and innovation related to wireless healthcare. Since continuous coverage of personal monitoring requires multiple systems, we identify the issues and gaps between systems, and we describe how Total Health could be achieved.

\section{Why Total Health Is Important?}

Healthcare costs are a major portion of the US gross domestic product, current estimates by OECD are approximately 17\% of GDP [4]. This is the highest in the world; most developed nations have their healthcare spending between $7-11 \%$ of GDP. Not only is the US healthcare spending at a very 
high level, it has been increasing since the beginning of the recorded data in 1960's. There are many reasons why this is so: the population is aging, utilization of health services is increasing, more advanced technology is used, labor resources are not increasing at the same rate as usage, insurance costs escalates with liability issues, and so healthcare is getting more expensive [5].

Chronic illness accounts for $78 \%$ of the total US healthcare spending [6]. From the 2004 US in-patient cost estimates on Medicare by the Agency for Health Quality Research, Healthcare Cost and Utilization Project, the five most costly of the chronic diseases in the US are: coronary artery disease (\$39.6 billion), heart failure ( $\$ 19.8$ billion), mental health disorders $(\$ 11.4$ billion), chronic obstructive pulmonary disease ( $\$ 8.2$ billion), and diabetes ( $\$ 7.4$ billion) [6. These staggering amounts lead many to believe that much of the costs are preventable.

Approximately $95 \%$ of the $\$ 1.4$ trillion that we spend as a nation on health goes to direct medical services, while approximately $5 \%$ is allocated to preventing disease and promoting health.

The vast majority of the expenses is being incurred on prolonged hospital stays and managed care after which the chronic conditions have progressed past any turning point. Only $5 \%$ is spent on prevention. If many of these conditions are prevented earlier in the cycle by detecting occurrences of key indicators or proactively monitoring high-risk patients, then a major portion of the healthcare costs will be eliminated. It is clear that chronic conditions currently are not being managed correctly. Even treatment at any earlier stage will reduce some of the costs.

Unfortunately, the current medical environment is one of reactive action and it is too late in many cases. By that point, the costs have escalated into the hundreds of thousands of dollars per patient. Some studies have shown up to $40 \%$ reduction in hospital admissions, $67 \%$ reduction in mortality and $62 \%$ reduction in bed days of care 8 .

One solution is to provide as much out of hospital care as possible. This could be in the form of trained health care providers that operate outside of the hospital and are focused on personalized home-based care. Unfortunately this would not address the ever increasing health bill, since the current labor force would be stretched thin and in turn require more and more people to be trained in healthcare. By adding more people, the total expense for healthcare would increase.

The other and more reasonable solution would be relying on technology. Technology can provide monitoring services not only within hospital environments but also at home. Current research has focused on many approaches to home-based remote monitoring. User initiated measurement devices such as weight scales and blood pressure monitors are one form of technology that have been wirelessly connected to monitoring systems. More ubiquitous systems such as those that are smartphone-based are also included in these solutions. These types of systems are not location dependent and professional 
medical help can be contacted when needed or, ideally, professional medical help will contact the patient when pending danger occurs.

Such Total Health systems are complementary to the traditional forms of healthcare services and are not intended to replace them. The overall goal is to improve the quality of healthcare while reducing the costs. To this end, the strategy of moving the healthcare process toward the patient for in-home care, relying more on the patient's own personal needs and control, actually promotes active, healthy living style. In the end, Total Health improves the overall quality of life.

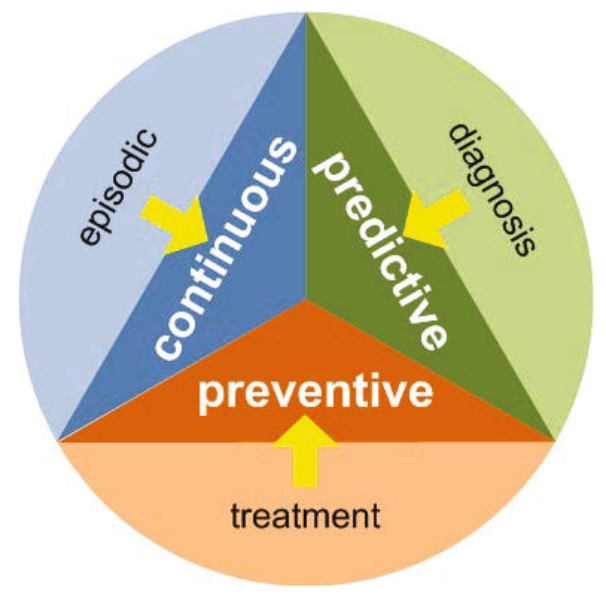

Fig. 1 Transformation of Healthcare Environment

Not only are there cost benefits and reductions in the need for ongoing medical interventions, but also these continuous systems improve the compliance of medical condition management. It is estimated that up to $\$ 100$ billion is wasted due to poor medical adherence [9]. Continuous health management systems can address some of the problems with non-compliance. With automatic analysis tools, pertinent data can also be presented to the user about health issues. Furthermore, mobile communication with healthcare providers can provide current monitored data as well as important alerts.

So, the goals of Total Health are:

- Use ubiquitous technology for sensing, monitoring, analyzing, and communicating to effectively manage healthy living.

- Be available $24 / 7$.

- Be as unobtrusive as possible. Be as comfortable as possible.

- Require as little human intervention as possible.

- Identify important indicators of possible health risk.

- Provide information to the user or medical providers about health alerts.

- Be cost effective. Reduce costly and unnecessary medical services that are common with current healthcare practices. 


\section{What Is the Impact?}

It is important to realize that the change to Total Health is a cultural and sociological change. It requires a change in people's thinking, a change in one's idea of healthcare control, and a change in responsibility. The current landscape of healthcare has a limited supply of healthcare providers and they are usally overwhelmed by the process of information collection. It is a reactive environment in which action is taken after significant events occur. However, a shift in the process will allow physicians to concentrate on providing the medical expert care at the right time [5]. Continuous remote monitoring of the patient takes much of the load away from doctors. The focus toward patient monitoring will relieve much of the work of the healthcare professional. It requires a paradigm change toward user responsibility and pre-emptive action. Continuous and ubiquitous healthcare systems provide a promising answer to today's medical questions.

The impact of Total Health is a three-fold transformation in the healthcare environment: (1) from episodic examination to continuous monitoring; (2) from disease diagnosis to disease prediction; (3) from reactive treatment to pre-emptive prevention. Figure 1 summarizes this change in healthcare. Currently episodic patient examination only captures snapshots of the health status of individuals. Total Health aims for continuous coverage of healthcare. Currently, a disease is only diagnosed if a patient has a health complaint and makes a visit to a healthcare professional. The focus of healthcare change aims to move the analysis toward prediction. Once conditions have been diagnosed in patients, reactive treatment plans are prescribed. However, often this is too late. We must strive for pre-emptive prevention of medical condition. There are existing steps toward pre-emptive prevention, such as early screenings for breast cancer and prostate cancer, however there is more that can be done.

\section{What Are the Challenges of Total Health?}

The goals of Total Health are ambitious and there are many factors that contribute to its future success. Bott et al. describe some of the challenges in bringing ubiquitous computing to healthcare [10]. Adams and Brown note some ethical issues involved in continuous monitoring of patients 11. Little and Briggs discuss real survey responses about the main concerns of shared personal data [12. In this section, we summarize some of the challenges of bringing Total Health to all, with the focus on technological issues.

Continuous patient monitoring generates a considerable amount of raw data which leads to storage problems and information overload. If the data is stored in the device, then it creates an issue of memory storage. If the data is to be sent remotely to physicians, then there is a huge demand on the communication process. Data size can be reduced by periodic sampling; however, 
this requires to be balanced with the continuous monitoring requirements of the system. Periodic sample will reduce both the local storage requirements of the device as well as communication requirements.

Other types of applications do not require raw data at all; instead, an analysis or summary of the data is used. In other applications, anomaly detection is done. In these situations, local device processing of the data is performed, and then the results are logged or transmitted.

Questions that are raised about data management are about how reliable and accurate the data process can be. Certainly errors do creep into systems, but how tolerant of errors should systems be? Issues such as the reliability of data and even of data transmission to physicians need to be considered by implementers and users of these systems. During medical emergencies, it is critical that systems can communicate with medical providers and also with time constraints. There are issues with mobility of systems. Ideal systems should work indoors and outdoors, as well as moving between mobile network cells.

One issue that could severely hamper the widespread adoption of remote monitoring is the health risk associated with the radiation effects of wireless technology. The World Health Organization and the International Agency for Research on Cancer has recognized that "radiofrequency electromagnetic fields as possibly carcinogenic to humans" 13. To this end, wireless technology aims at lowering the power consumption of mobile communication and has the benefit of improving the battery life of the mobile devices.

One of the major challenges in remote mobile devices is the use of conventional batteries which are heavy, rigid, and do not last long. More research needs to be done in developing batteries which are light, flexible, and easily chargeable [14. The transformation toward pro-active monitoring requires better power supply management and longer battery life.

In all of the above situations, there is always the consideration of costs to implement and costs to maintain. We have discussed the need to reduce the overall healthcare spending, but it mandates that the change in technology and labor will be low cost. This is the assumption, however, there is no upto-date reports on real financial improvements.

The rate of adoption to change in technology is remarkably slow in the medical industry. Compared to banking, such as in online account management, electronic medical records have been slow to gain widespread use. Healthcare has lagged behind other industries in electronic records keeping. There are many reasons for this. The cost of moving to electronic data management is high and only larger institutions are able to absorb the costs. Private practices and small communities cannot justify the expenditure. Healthcare workflow is not easily standardized or amenable to electronic formats. So, the healthcare environment is possibly set for a dramatic change in lifestyle. 


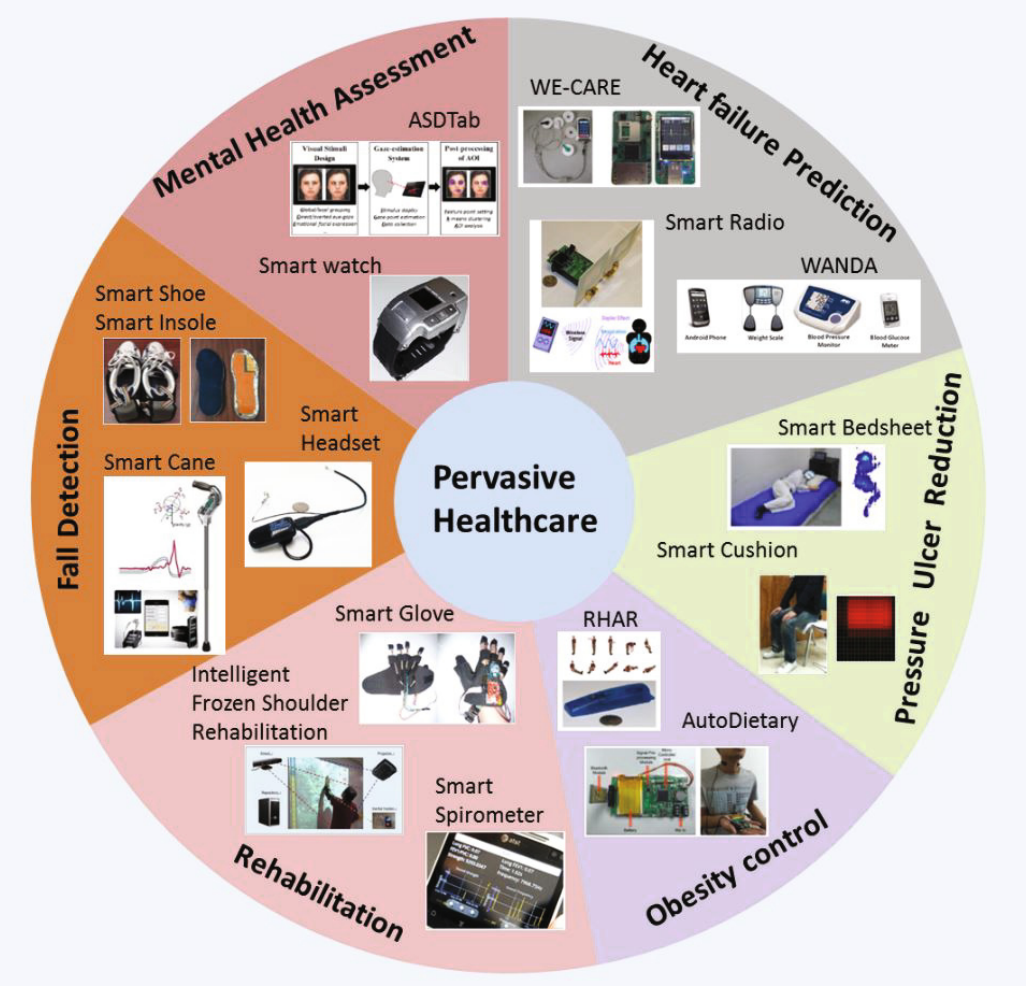

Fig. 2 Applications of Total Health

\section{Total Health Applications}

In this section, we detail a selection of medical applications where continuous monitoring systems will affect the change toward better human health. There are many more applications that will see huge benefits that are not described here. Figure 2 shows five applications that have seen promising system developments: heart failure, pressure monitoring, fall detection, mental health assessment, and applications for rehabilitation.

Within each application, there could be a number of systems that work together to provide complete coverage. Each of the systems can be broadly categorized [15] into:

- Mobile devices: devices that are portable such as smart phones, tablets, PDAs, and even laptops.

- Wearable items: devices that can be a part of clothing or e-textile, or devices that are strapped to the body.

- Stationary devices: devices such as sensors embedded or mounted in furniture, building walls, or fixed structures. 
Although there has been a lot of development and good applications for implantable devices, such as pacemakers, defibrillators, diabetic implants, we shall not include such devices in this paper due to their invasiveness.

The following subsections introduce current development as well as relevant applications for the technology. We also identify the challenges in bringing the systems to widespread use. No one system can effectively satisfy all the requirements of Total Health. A combination of systems would work to complement each other. We also discuss the gaps and overlaps in the systems.

\subsection{Heart Failure Prediction}

Cardiopulmonary signals are the most important human biomarkers and have many important medical implications, especially for chronic health conditions such as heart diseases, chronic obtrusive pulmonary disorders (COPD) disease and diabetes. A significant portion of that cost comes from the expense of monitoring patients and transferring the recorded data to physicians.

A technology that is currently being investigated is a Doppler radar to measure human vital signs [16]. It is a non-contact autonomous vital sign monitoring system, called Smart Radio (Figure 3). Based on Doppler theory, this economic and lightweight Doppler-radar system senses chest wall movement and heartbeat, which are highly correlated to respiratory rate and heart rate. This enables wireless vital sign measurements that are completely unobtrusive and can pass through clothing. Compared to traditional vital sign measurement instruments, Smart Radio is easier to deploy and more convenient to use due to the non-contact sensing and auto-calibration scheme.

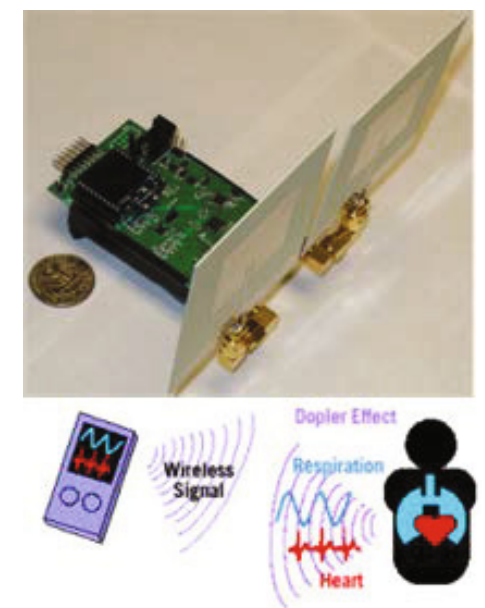

Fig. 3 Smart Radio 16 
Cardiovascular disease is the No. 1 cause of death in the past 30 years. Longitudinal monitoring on heart status is important for heart failure patients. WE-CARE [17)(see Figure 4) is an online risk-monitoring systems which measures ECG signals to offer real-time risk monitoring. For the sake of the sustainability of use, a compressed sensing based technique is fed into the system to reduce power consumption. This research product has been approved by Ministry of Health $(\mathrm{MOH})$ in China and under evaluation by thousands of heart failure patients.

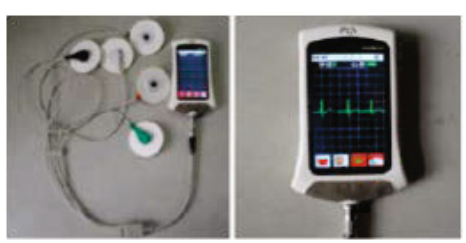

Fig. 4 WE-CARE 17

Suh et al. developed a remote patient monitoring system called WANDA to monitor subjects post-surgically who had suffered congestive heart failure [18. The system integrates weight scales, blood pressure monitors, blood glucose meters, and cell phones via Bluetooth (Figure 5). Once all patient data has been measured, the information is communicated automatically over cell phone networks to servers located on the Internet where the data is analyzed by health professionals. Complete history of the patients is stored in databases and any anomalies can be detected and the patient notified immediately. Many of the large medical device companies are offering their own versions of remote patient monitoring such as Philips' Telestation, Bosch's Health Buddy, and Honeywell's Genesis DM.

The systems do rely on daily action by the patient, but give them reminders to use the monitors when overdue. The cost benefit is realized when a single medical provider can monitor a group of patients under their care. Early detection of problems is referred to specialized care before major problems

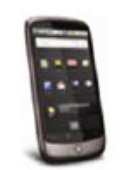

Android Phone

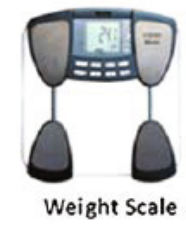

Weight Scale

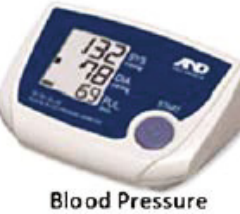

Monitor

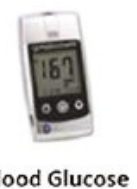

Meter

Fig. 5 WANDA 18 
occur and avoid expensive re-hospitalizations. The process of remote patient monitoring is becoming increasingly used and the range of applications is expanding from heart monitoring (e.g. chronic heart disease) to diabetes, hypertension, and lung disease (e.g. chronic obstructive pulmonary disease).

An important part of these systems is feedback and education. It is generally known that feedback of progress is a good method of continued encouragement. These systems also provide education through multimedia and online coaching. The current trend is availability of information. All of the above systems have methods to communicate data into cloud networks typically through cell phone networks to the Internet. Data can be stored in personalized electronic health record systems that can provide a complete longitudinal record of the patient's medical history. Medical professionals to provide the best ongoing healthcare can access this information, if they obtain required authorization.

\subsection{Pressure Ulcer Reduction}

E-textile sensors can record biometric data during the day while patients wear such devices as WE-CARE, and at night they are monitored by Doppler technology in their beds, this would be a good step toward Total Health. The gaps could be the current ECG recording requires direct skin contact sensors, so further research needs to be done in finding non-contact ECG recording. In fact, non-skin contact sensors have been developed [19. These sensors can be attached to clothing and can detect changes in the electrical field around the patient. The sensors need not be attached directly to the skin. This one significant advance toward Total Health since placing sensors on clothes is less obtrusive that directly on the skin.

E-Textile sensors applied in Smart Cushion system for dedicated underhip pressure analysis while sitting. Xu et al. 20] developed a dynamic time warping based algorithm to recognize different sitting postures base on Smart Cushion.

Sleep plays a pivotal role in the quality of life, and sleep posture is related to many medical conditions such as pressure ulcers. A pressure-sensitive bed sheet system provides an unobtrusive method for sleep posture monitoring and sleep analysis 21. Further application is on-bed physical rehabilitation for those who are recovering from surgery or for the bed prone.

Although this system is not mobile, it is hoped to be cost effective enough for it to be widely distributed, less than $\$ 1,000$. For comfort the bed sheet feels like a regular bed sheet, and does not require wearable devices for monitoring. The combination of radar-based vital sign measurement, Smart Radio, and pressure sensitive bed sheet enables comprehensive monitoring of patients during their sleep. 


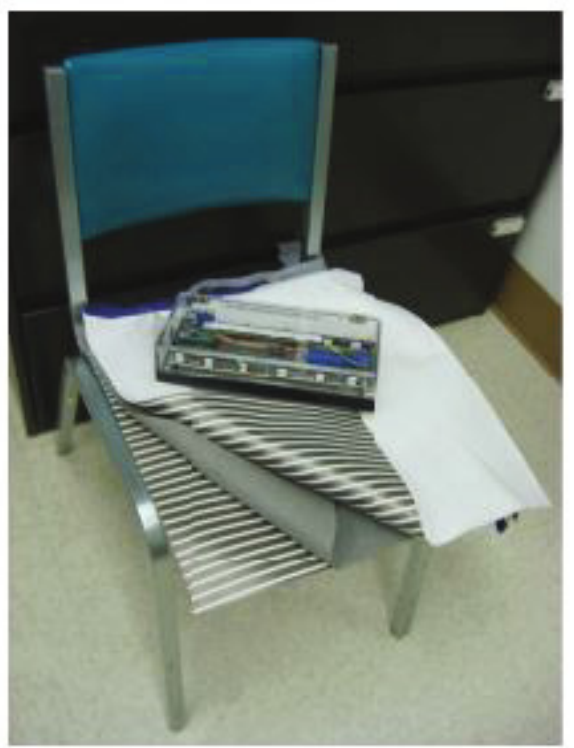

Fig. 6 Smart Cushion

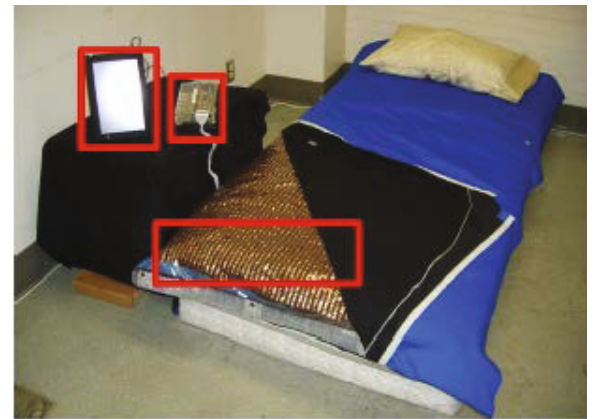

Fig. 7 Smart Bedsheet

\subsection{Fall Detection}

Annually, 1.8 million older adults are treated for falls, which results in $\$ 20$ billion in direct costs in 2008 [22, 23. A lightweight smart shoe and supporting infrastructure aims at gait and human balance monitoring. This system comprises accelerometers, compass, and pressure sensors mounted within shoe insoles with built-in Bluetooth communication to cell phones [24, 25, 26]. This system monitors walking behavior and uses instability assessment to classify various types of activity. Due to the challenge power consumption, an energy-efficient adaptive sensing was proposed which can effectively reduce the number of samples required per frame and still keep good signal quality 


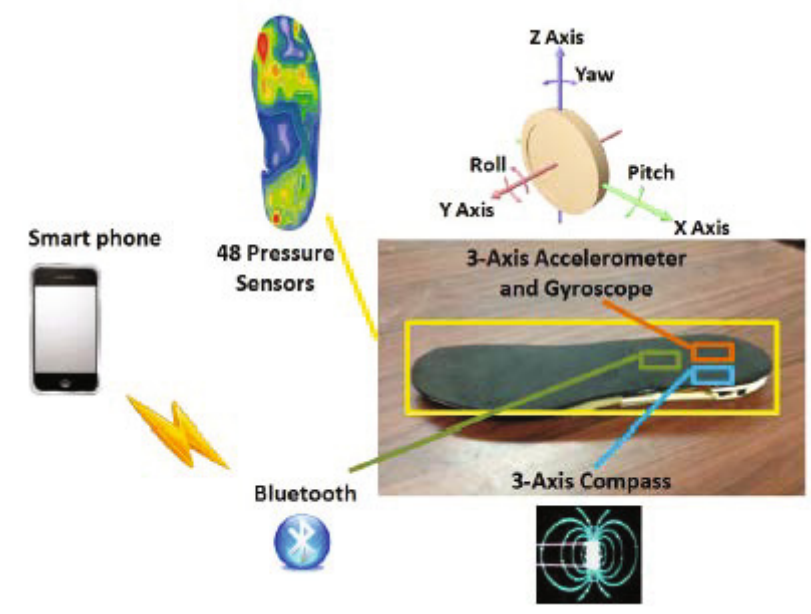

Fig. 8 Smart Insole

and gait parameters 26]. An important application of this technology is to determine fall risk and identification of episodes as precursors to instability. For mainstream use, the cost of smart insoles need to be viable, currently the estimated manufacturing cost is under $\$ 200$ with further research being continued.

For applications, such as activity monitoring, the smart insole captures a record of a person's activity. This is beneficial for obesity analysis and general fitness. Other applications are pressure ulcers that develop especially for diabetics. The smart insole can analyze the pressure distribution of a person's foot and generate alerts for prolonged pressure points.

Smart Headset is a lightweight EEG signal sensing and analysis devices, and it can calculate the level of concentration of users while walking. Intelligence also has been built into an assistive device like the smart cane [27. The smart cane is design to monitor and predict situations of high falling risk. The same technology is employed in smart crutches. Having to use crutches does not occur frequently for most people, and the proper use of crutches requires a balanced amount of pressure. Pressure sensors can give the best feedback to the user.

\subsection{Mental Health Assessment}

Autism spectrum disorder (ASD) is a neurodevelopmental disorder defined by atypicalities in three major symptom domains: communication, social interaction and behavior 28. Children with ASD have impaired social interaction, impaired verbal and non-verbal communication, and restricted, repetitive or stereotyped behavior, which may result in lifelong disabilities. These disorders 


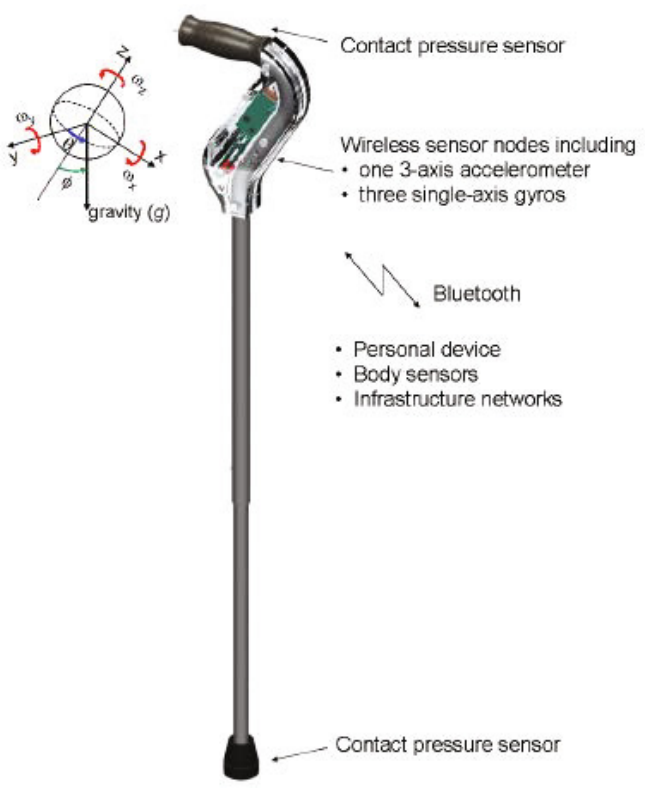

Fig. 9 Smart Cane 27]

both across and within individuals present particular challenges for autism diagnosis and treatment. ASDTab, a novel in-home ASD assessment tool, mainly comprises three parts, as show in Figure 15. (1) Visual Stimuli Design, (2) Gaze Estimation System and (3) Data Post-processing. ASDTab implements a highly-accurate eye-tracking and collects the gaze points of the child which means the impression of the interest areas of the child, it is important for ASD diagnosis.

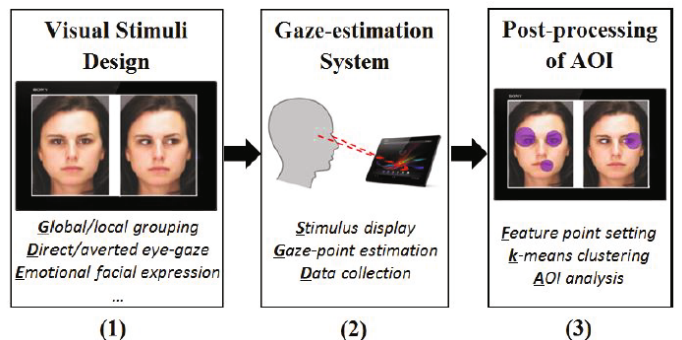

Fig. 10 ASDTab system mainly involves three parts: (1) Visual Stimuli Design; (2) Gaze-point Estimation; (3) AOI (area-of-interest) Detection 


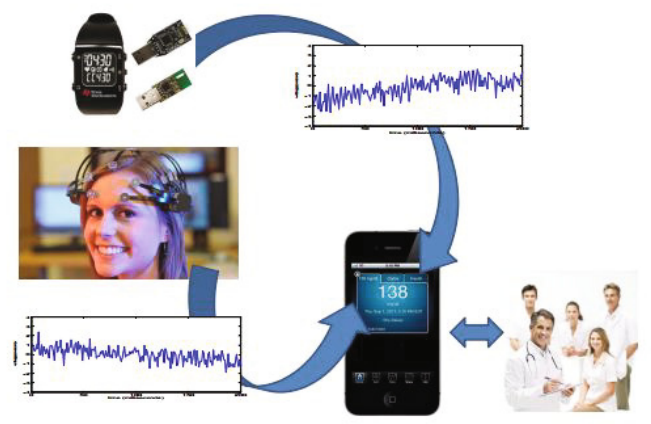

Fig. 11 Smartphone Monitoring for Management of Parkinson's Disease 29]

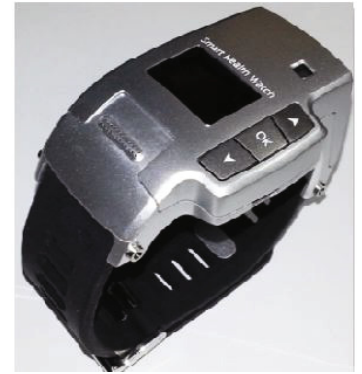

Fig. 12 Smart Watch for Dementia Patients 30.

Parkinson's Disease (PD) is a disabling neurodegenerative disease affecting millions of people worldwide. Teresa et al. 29] developed a system for portable monitoring of Parkinsonism based on EEG signal features and advanced techniques for monitoring human movement from gyroscopes and accelerometers.

Dementia means cognitive impairment that makes functioning in daily life more difficult in the older population. A Smart Watch System was developed for dementia patients to improve their health and safety [30]. The smart watch system includes a wristwatch-type device and server system. The device includes a built-in GPS, ambient light sensor, acceleration sensor, and to communicate with the server system. The system can create a personal profile for patients and monitor the location, motion through the amount of light and step count.

\subsection{Obesity Control}

Nutrition-related diseases are nowadays a main threat to human health and pose great challenges to medical care. A crucial step to solve the problems 

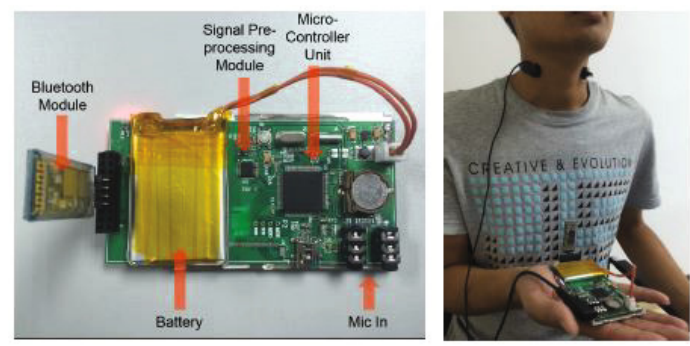

Fig. 13 AutoDietary 31]
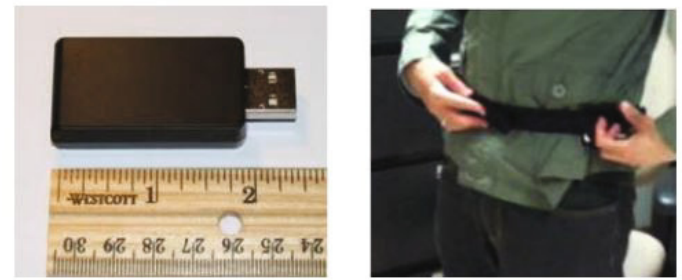

Fig. 14 Motion sensor for Activity Recognition 32

is to monitor the daily food intake of a person precisely and conveniently. AutoDietary [31] (see Figure 13), a wearable system to monitor and recognize food intakes in daily life, is developed to collect food intake sensor data, which is highlighted by a high-fidelity microphone worn on the subject's neck to precisely record acoustic signals during eating in a non-invasive manner. The corresponding application on the smartphone which aggregates the food intake recognition results in a user-friendly way and provides suggestions on healthier eating, such as better eating habits or nutrition balance.

Wearable sensors, specifically inertial sensors, continue to be used in activity recognition systems and devices. Alshurafa et al. 32 designed a robust activity recognition framework for health and exergaming using wearable sensors. The application is a novel exergaming environment aimed at using games to reward physical activity performed throughout the day, to encourage a healthy lifestyle.

\subsection{Rehabilitation}

People with stroke often need physical therapy services to improve stroke symptoms or retain functionality and independence with daily activities. SmartGlove consists of five-finger bending sensors and is a finger angle extraction device which is packaged in an easy-to-wear and adjustable manner for a patient with an upper extremity rehabilitation progress [33. 


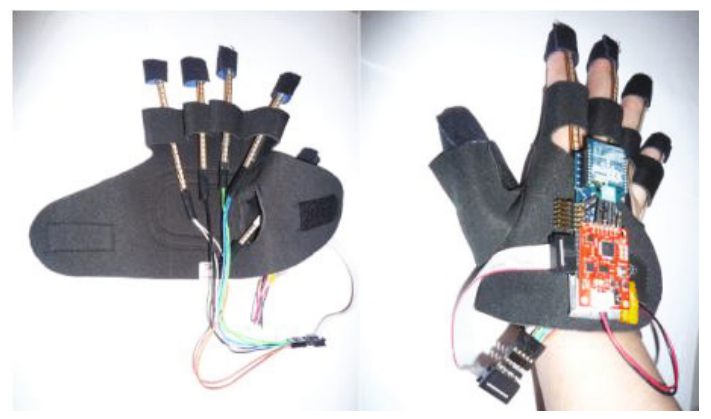

Fig. 15 Smart Glove 33

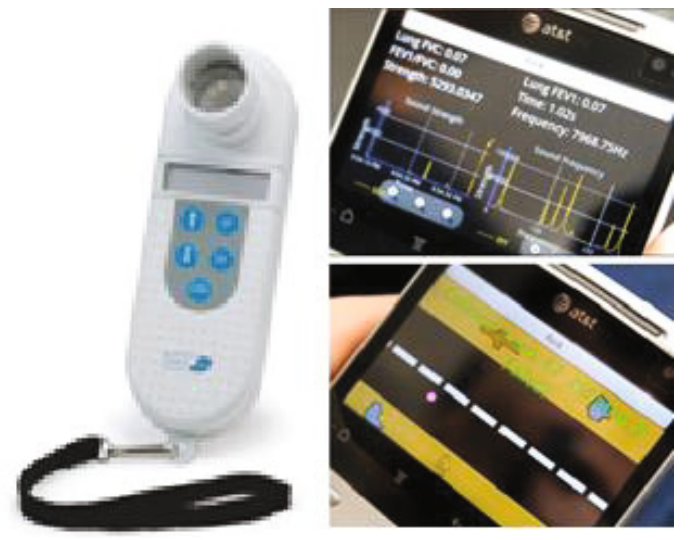

Fig. 16 Smart Spirometer 34]

Smart Spirometer (See Figure [16) is a mobile-phone based platform to measure lung function parameters (FEV1, FVC, PEF), which are critical clinic evidences for CPOD, asthma, and cystic fibrosis diagnosis. Taking advantage of the existing built-in microphone in every phone, this system modeled the microphone frequency-domain response on airflows and has the function of accurate lung function measurement. Moreover, to increase patient-compliance for breathing exercise, this system has a phone video game for daily respiration exercise and training 34 .

Frozen shoulder is a common condition characterized by painful and limited range of motion. Using interactive technologies can help patients complete the exercises crucial to their rehabilitation. Huang et al. 35] created a virtual reality game-based treatment system that encourages patients to participate in regular rehabilitation. Vision and inertial sensors are used to collects patient ROM data during the game tasks for further analysis.

For people with imbalance and vestibular dysfunction issues, this study used VR video games that adopted Cawthorne\&Cooksey exercises and machine learning-based classifiers as a balance assessment tool [36]. The objective of this 


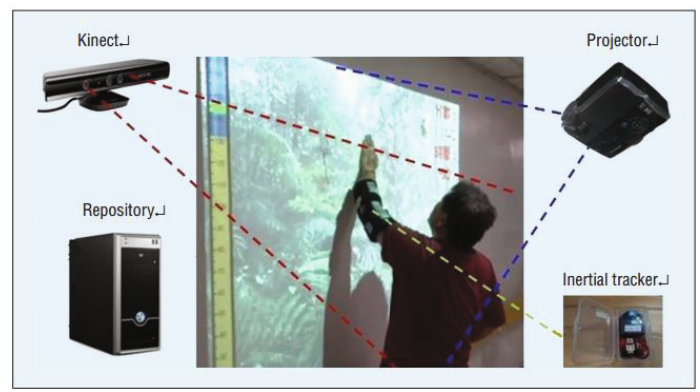

Fig. 17 Intelligent Frozen Shoulder Rehabilitation System 35.

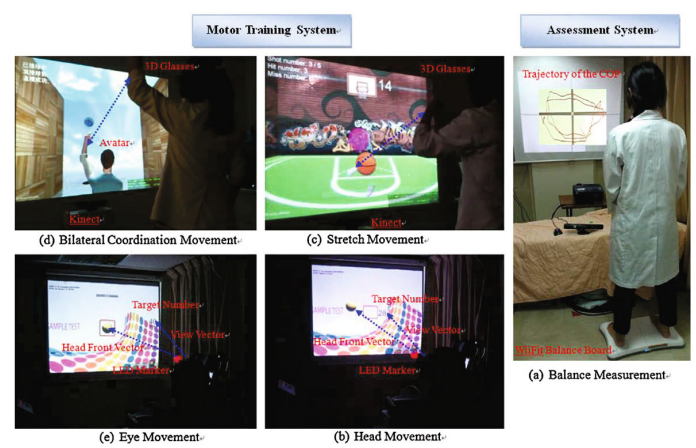

Fig. 18 Imbalance and Vestibular Dysfunction Rehabilitation System 36

study is to validate a VR system that can be used for balance rehabilitation. The consistent findings between the performance data and balance assessment measurements suggest that this VR game-based exercise may be applicable to the clinical assessment and rehabilitation of people with vestibular dysfunction.

\section{Conclusion}

This paper presented the concept of Total Health. It is clear that complete health monitoring is the way forward. In fact, remote patient monitoring devices had the fastest growth in yearly revenue of any medical device sectors and the US market will be worth $\$ 20$ billion in 2016 [37. The future of healthcare will see a boom in patient-controlled out-of-hospital monitoring, with personal health data shared with the patient's healthcare provider. There is much hope that continuous and ubiquitous healthcare systems will supply a solution for today's healthcare situation. However, this will require a transformation of the current concept of healthcare. 


\section{References}

1. Eysenbach, G.: What is e-health? J. Med. Internet Res. 3(2), 20 (2001)

2. Krupinski, E., Nypaver, M., Poropatich, R.: Telemedicine/telehealth: An international perspective. clinical applications in telemedicine/telehealth. Telemed. J. E Health 8(1), 13-34 (2001)

3. Weiser, M.: The computer for the 21st century. SIGMOBILE Mob. Comput. Commun. Rev. 3(3) (July 1999)

4. Organisation for economic co-operation and development (2012), http://www. oecd.org/

5. Enabling ubiquitous patient monitoring: Model, decision protocols, opportunities and challenges. Decision Support Systems 46(3), 606-619 (2009)

6. I. E.-H. Committee, Chronic care improvement: How medicare transformation can save lives, save money, and stimulate an emerging technology industry. ITAA E-Health White paper (2004)

7. Steps to a healthier u.s. washington (dc): Office of public health promotion. U.S. Department of Health and Human Services (2001), http://www.healthierus.gov/steps/steps_brochure.pdf

8. Dang, S., Dimmick, S., Kelkar, G.: Evaluating the evidence base for the use of home telehealth remote monitoring in elderly with heart failure. Telemedicine and e-Health 15, v (2009)

9. Cocosila, M., Archer, N., Yuan, Y.: Mobile healthcare for adherence: Business considerations. Mednet, Toronto, Canada, pp. 13-20 (October 2006)

10. Bott, O., Ammenwerth, E., Brigl, B., Knaup, P., Lang, E., Pilgram, R., Pfeifer, B., Ruderich, F., Wolff, A., Haux, R., Kulikowski, C.: The challenge of ubiquitous computing in health care: technology, concepts and solutions. findings from the imia yearbook of medical informatics. Methods Inf. Med. 44(3), 473-479 (2005)

11. Adams, A.A., Brown, I.: The ethical challenges of ubiquitous healthcare. International Review of Information Ethics 8, 53-60 (2007)

12. Little, L., Briggs, P.: Ubiquitous healthcare: do we want it? In: Proceedings of the 22nd British HCI Group Annual Conference on People and Computers: Culture, Creativity, Interaction, BCS-HCI 2008, Liverpool, United Kingdom, vol. 2, pp. 53-56 (2008)

13. World health organization/iarc (2011) http://www.iarc.fr/en/media-centre/pr/2011/pdfs/pr208_E.pdf

14. Khosla, A.: Smart garments in chronic disease management: progress and challenges. In: Proc. SPIE 8548, Nanosystems in Engineering and Medicine, pp. 85 482O-85 482O-8 (2012)

15. Orwat, C., Graefe, A., Faulwasser, T.: Towards pervasive computing in health care - a literature review. BMC Medical Informatics and Decision Making 8(1), $26(2008)$

16. Huang, M.-C., Liu, J.J., Xu, W., Gu, C., Li, C., Sarrafzadeh, M.: A selfcalibrating radar sensor system for measuring vital signs. IEEE Transactions on Biomedical Circuits and Systems (2015)

17. Huang, A., Xu, W., Li, Z., Xie, L., Sarrafzadeh, M., Li, X., Cong, J.: System light-loading technology for mhealth: Manifold-learning based medical data cleansing and clinical trials in we-care project. IEEE Journal of Biomedical and Health Informatics (JBHI) 18(5), 1581-1589 (2014) 
18. Suh, M.-K., Chen, C.-A., Woodbridge, J., Tu, M., Kim, J., Nahapetian, A., Evangelista, L., Sarrafzadeh, M.: A remote patient monitoring system for congestive heart failure. Journal of Medical Systems 35, 1165-1179 (2011)

19. Chi, Y., Cauwenberghs, G.: Wireless non-contact eeg/ecg electrodes for body sensor networks. In: 2010 International Conference on Body Sensor Networks (BSN), pp. 297-301 (June 2010)

20. Xu, W., Huang, M.-C., Amini, N., He, L., Sarrafzadeh, M.: ecushion: A textile pressure sensor array design and calibration for sitting posture analysis. IEEE Sensors Journal 13(10), 3926-3934 (2013)

21. Liu, J.J., Xu, W., Huang, M.-C., Alshurafa, N., Sarrafzadeh, M.: A dense pressure sensitive bedsheet design for unobtrusive sleep posture monitoring. under review IEEE International Conference on Pervasive Computing and Communications (2013)

22. Centers for disease control and prevention, http://www.cdc.gov/HomeandRecreationalSafety/Falls/fallcost.html

23. Stevens, J., Corso, P., Finkelstein, E., Miller, T.: The costs of fatal and nonfatal falls among older adults. Injury Prevention 12, 290-295 (2006)

24. Noshadi, H., Ahmadian, S., Hagopian, H., Woodbridge, J., Dabiri, F., Amini, N., Sarrafzadeh, M., Terrafranca, N.: Hermes - Mobile Balance and Instability Assessment System. In: Proc. BIOSIGNALS, pp. 264-270 (2010)

25. Xu, W., Huang, M., Amini, N., Liu, J., He, L., Sarrafzadeh, M.: Smart insole: A wearable system for gait analysis. In: PETRAE (2012)

26. Xu, W., Wu, Y., Liu, J., Huang, M., Luan, S., Lee, Y.: An energy-efficient adaptive sensing framework for gait monitoring using smart insole

27. Wu, W., Au, L., Jordan, B., Stathopoulos, T., Batalin, M., Kaiser, W., Vahdatpour, A., Sarrafzadeh, M., Fang, M., Chodosh, J.: The smartcane system: an assistive device for geriatrics. In: Proceedings of the ICST 3rd International Conference on Body Area Networks, BodyNets 2008 (2008)

28. Wing, L., Gould, J.: Severe impairments of social interaction and associated abnormalities in children: Epidemiology and classification. Journal of Autism and Developmental Disorders 9(1), 11-29 (1979)

29. Sanders, T.H., Devergnas, A., Wichmann, T., Clements, M.A.: Remote smartphone monitoring for management of parkinson's disease. In: Proceedings of the 6th International Conference on PErvasive Technologies Related to Assistive Environments, p. 42. ACM (2013)

30. Shin, D.-M., Shin, D., Shin, D.: Smart watch and monitoring system for dementia patients. In: Park, J.J.(J.H.), Arabnia, H.R., Kim, C., Shi, W., Gil, J.-M (eds.) GPC 2013. LNCS, vol. 7861, pp. 577-584. Springer, Heidelberg (2013)

31. Bi, Y., Xu, W., Guan, N., Wei, Y., Yi, W.: Pervasive eating habits monitoring and recognition through a wearable acoustic sensor. In: 8th International Conference on Pervasive Computing Technologies for Healthcare (PervasiveHealth 2014), Oldenburg, Germany, pp. 1-4 (May 2014)

32. Alshurafa, N., Xu, W., Liu, J.J., Huang, M.-C., Mortazavi, B.J., Sarrafzadeh, M.: Designing a robust activity recognition framework for health and exergaming using wearable sensors. IEEE Journal of Biomedical and Health Informatics (JBHI) 18(5), 1636-1646 (2014)

33. Huang, M.-C., Xu, W., Su, Y., Lange, B., Chang, C.-Y., Sarrafzadeh, M.: Smartglove for upper extremities rehabilitative gaming assessment. In: Proceedings of the 5th International Conference on Pervasive Technologies Related to Assistive Environments, p. 20. ACM (2012) 
34. Xu, W., Huang, M.-C., Liu, J.J., Ren, F., Shen, X., Liu, X., Sarrafzadeh, M.: Microphone-based spirometer for copd diagnosis and exergame design. In: International Conference on Pervasive Technologies Related to Assistive Environments (PETRA 2013), Rhodes Island, Greece, pp. 45-52 (May 2013)

35. Huang, M.-C., Lee, S.-H., Yeh, S.-C., Chan, R.-C., Rizzo, A., Xu, W.: Intelligent frozen shoulder rehabilitation using virtual reality

36. Machine learning-based assessment tool for imbalance and vestibular dysfunction with virtual reality rehabilitation system. Computer Methods and Programs in Biomedicine 116(3), 311-318 (2014)

37. Kalorama information,

http://www.kaloramainformation.com/Remote-Wireless-Patient-6487095/ 